\title{
EVN observations of candidate Compact Symmetric Objects
}

\author{
L. Xiang ${ }^{1}$, C. Stanghellini ${ }^{2}$, D. Dallacasa ${ }^{3,4}$, and Z. Haiyan ${ }^{5}$ \\ 1 National Astronomical Observatories, Chinese Academy of Sciences, Urumqi 830011, PR China \\ e-mail: liux@ms.xjb.ac.cn \\ 2 Istituto di Radioastronomia del CNR, C.P. 141, 96017 Noto SR, Italy \\ 3 Istituto di Radioastronomia del CNR, via P. Gobetti 101, 40129 Bologna, Italy \\ 4 Dipartimento di Astronomia, Alma Mater Studiorum, via Ranzani 1, 40127 Bologna, Italy \\ e-mail: ddallaca@ira.cnr.it \\ 5 National Astronomical Observatories, Chinese Academy of Sciences, Beijing 100012, PR China \\ e-mail: hyzhang@class1.bao.ac.cn
}

Received 14 September 2001 / Accepted 28 January 2002

\begin{abstract}
We present pc-scale images of ten Compact Symmetric Objects (CSO) candidates observed with the European VLBI network (EVN). Five radio sources have been observed at $1.6 \mathrm{GHz}$, and five more at 2.3/8.4 GHz, the latter subsample with the inclusion in the VLBI array of 3 antennae normally used for geodesy. These objects were selected from existing samples of $\mathrm{GHz}$ Peaked Spectrum (GPS) radio sources with the purpose to find and/or confirm the CSO classification. These new VLBI observations allow us to confirm the classification of two CSO candidates, and to find a few new ones. The association of GPS radio galaxies with a CSO morphology is strengthened by our findings, and this result suggests an efficient way to increase the number of known CSOs by means of VLBI observations of compact radio galaxies showing a convex radio spectrum.
\end{abstract}

Key words. galaxies: active - quasars: general - radio continuum: galaxies

\section{Introduction}

Compact Symmetric Objects (CSOs) form a class of radio sources with distinctive radio properties. They are very powerful and compact sources with overall size $<1 \mathrm{kpc}$, dominated by lobe/jet emission on both sides of the central engine, and are thought to be relatively free from beaming effects (Wilkinson et al. 1994). Their small size is likely due to their youth $\left(<10^{4} \mathrm{yr}\right)$. This hypothesis has been largerly accepted after the detection and the estimate of the separation speed of the micro hot-spots in a couple of CSOs (Owsianik \& Conway 1998; Owsianik et al. 1998). Since then, expansion velocities of the outer edges have been detected or suspected in a handful of other CSOs (Fanti 2000 and references therein).

A unification scenario assumes that CSOs evolve into Medium-size Symmetric Objects (MSO), which, in turn, evolve into Large Symmetric Objects (LSO), i.e. large FRII radio sources (Fanti et al. 1995; Readhead et al. 1996a,b; Snellen et al. 2000). The monitoring of the structural changes over time seems one of the more promising ways to understand the evolution of these objects, and a

Send offprint requests to: C. Stanghellini,

e-mail: cstan@ira.cnr.it larger number of CSOs suitable for these repeated observations is desirable.

Most confirmed CSOs and CSO candidates are commonly found to have a global convex radio spectrum peaking at $\mathrm{GHz}$ frequencies, thus belonging to the class of the GHz Peaked Spectrum (GPS) radio sources.

These latter objects are known to show structures resolved only with VLBI. Only about $10 \%$ of them shows faint extended radio emission on scales of a few tens of kpc, while $\sim 90 \%$ of them is entirely contained within the extent of the narrow-line region $(<1 \mathrm{kpc})$ of the host galaxy (Stanghellini et al. 1998). GPS radio sources are usually identified with galaxies at low or intermediate redshifts $(z<1)$, and quasars at higher redshifts (O'Dea et al. 1991; Snellen et al. 1999), and they make up a significant fraction $(\sim 10 \%)$ of the bright centimeter wavelength selected radio sources.

Many GPS radio sources have no optical counterpart yet, and these empty fields are most likely distant galaxies, too faint to be detected. Stanghellini et al. (1997a, 1998) find a strong correlation between the milliarcsecond (mas) morphology and the optical host in bright GPS sources. Galaxies are generally associated with CSOs while quasars have more often core-jet or complex morphology. 
There are a few tens of sources in known GPS samples, and many more are in candidate lists; several of these sources have either no or poor VLBI images. In order to increase the number of known CSOs, we selected from the lists of known bright GPS samples (O'Dea et al. 1991; de Vries et al. 1997; Stanghellini et al. 1998; Dallacasa et al. 2000) the sources accessible to the EVN without enough structural information in the literature to permit a proper classification yet. Scheduling the radio sources to be observed in the allotted time we gave a higher priority to objects with known double morphology at a single frequency.

We report here the results of the two first observing runs. In a first session at $1.6 \mathrm{GHz}$ with the EVN and MERLIN we observed 5 sources. At this frequency we could detect the presence of extended steep spectrum emission, determine the total angular size of the source, and possibly determine the low frequency spectral shape of the components, when other images at higher frequencies were available. Five further radio sources were observed at $2.3 / 8.4 \mathrm{GHz}$ with the EVN plus 3 antennae commonly used in geodesy experiments. These simultaneous observations at 2 frequencies are best suited to detect the cores.

\section{Observations and data reduction}

\subsection{EVN + MERLIN at $1.66 \mathrm{GHz}$}

EVN Observations were carried out in two separate VLBI experiments. The first observation has been done on 13 November 1999 at $1.66 \mathrm{GHz}$ using the MKIII recording system, with a total bandwith of $28 \mathrm{MHz}$, in left circular polarization, with the antennae of Effelsberg, Westerbork, Jodrell, Cambridge, Onsala, Medicina, Noto, Torun, Nanshan and Sheshan. Snapshot observations for a total of 5.2 hours of observing time were made for the sources $1345+125,1404+286$ (OQ208), 1518+047, $1604+315$, and $1751+278$. The MERLIN array simultaneously observed those sources with 7 antennae (Defford, Cambridge, Knockin, Darnhall, MK2, Lovell and Tabley) at the same frequency. The EVN $1.66 \mathrm{GHz}$ data were correlated at the MPIfR MKIII correlator.

The MERLIN data were calibrated in a standard way and the images have been made using the DIFMAP package (Shepherd 1997). We only show the MERLIN image of $1345+125$ as the other objects are pointlike at the resolution of about 150 mas. The MERLIN images of the remaining sources are shown in Zhang et al. (2001).

\section{2. $E V N+G E O$ at 2.3 and $8.4 \mathrm{GHz}$}

The second observation has been done on 29 May 2000 at 2.3/8.4 GHz using the MKIV recording system with a bandwith of $16 \mathrm{MHz}$ in each frequency, in right circular polarization. The EVN antennae involved in this experiment which gave useful data were Effelsberg, Westerbork, Onsala, Medicina, Noto, Yebes, plus the geodetic antennae of Matera, Ny-Alesund, Wettzel. Nanshan and Sheshan failed to give fringes because of problems in their recent upgrade to MKIV formatting system. Snapshot observations for a total of 7.9 hours of observing time were taken for $1333+589,1427+109,1509+054,1526+670$, and $1734+508$. The $2.3 / 8.4 \mathrm{GHz}$ data were correlated at the MKIV correlator at JIVE. This is one of a very few EVN experiment making use of several geodetic antennae, and their inclusion in the array has proven to be very useful with a major improvement in the NS resolution and UV coverage.

The Astronomical Image Processing System (AIPS) developed by the National Radio Astronomy Observatory (NRAO), has been used for editing, a-priori calibration, fringe-fitting, imaging and self-calibration. Several iterations of phase self-calibration were performed until we converged to an acceptable solution. Then one or more iterations of amplitude and phase-calibration were applied. We also used the IBLED and CLIP tasks in AIPS for editing the data before the first phase-calibration and/or after the first amplitude calibration because of the dispersion of the data on some baselines. The main source of errors of the flux densities estimated in our final VLBI images is the absolute flux density scale which is assumed to have uncertainties of $\sim 10 \%$.

The sizes and the flux densities of the components given in Tables 2 and 3 have been obtained with a Gaussian fit using the task JMFIT in AIPS.

\section{Results and comments on individual sources}

In this section we present the results from our VLBI observations. Basic information on the target sources is presented in Table 1. Tables 2 and 3 summarize the parameters derived from the images. In the following we present each source and comment on the results of the present observations.

\subsection{Images at $1.66 \mathrm{GHz}$}

\subsection{1. $1345+125(J 1347+1217,4 C 12.50)$}

$1345+125$ at $z=0.122$, is one of the nearest bright GPS radio sources. The radio source is associated with a galaxy $\left(m_{r}=15.5\right)$ lying at the center of a group. The optical image shows 2 well-resolved nuclei separated by $2^{\prime \prime}(4 \mathrm{kpc})$, with the radio source associated with the westernmost one (Stanghellini et al. 1993; Evans et al. 1999). The double nucleus, the irregular isophotes, the presence of a tail, and a number of close smaller companions indicates that $1345+125$ is likely to be in process of a merging, which had triggered the radio activity (Heckman et al. 1986).

The $5 \mathrm{GHz}$ radio image (Stanghellini et al. 1997a) shows a well defined structure visible in Fig. 1a, where component $\mathrm{A}$ is tentatively identified as the core, $\mathrm{B}$ and $\mathrm{C}$ are prominent knots in a jet, $\mathrm{D}$ is a mini-lobe, and $\mathrm{E}$ is tentatively identified as a much weaker counter lobe or jet. The whole radio emitting region is confined in less than 150 pc. Our image is the first $1.66 \mathrm{GHz}$ VLBI image of $1345+125$. We can identify the jet components (B, C), 
Table 1. GPS sources. Columns 1 through 13 provide: name, optical identification, optical magnitude, redshift (those with * are photometric estimates by Heckman et al. 1994), linear scale factor pc/mas $\left[H_{\mathrm{o}}=100 \mathrm{~km} \mathrm{~s}^{-1}\right.$ and $q_{\mathrm{o}}=0.5$ have been assumed], maximum VLBI angular size, maximum VLBI linear size, flux density at $1.6 \mathrm{GHz}$ as measured by the MERLIN, flux density at 1.6, 2.3, 8.4 GHz as measured from our VLBI images, low frequency spectral index, high frequency spectral index, computed turnover frequency, and references for the spectrum information (we use $S \propto \nu^{-\alpha}$ ): [1] Stanghellini et al. (1998), [2] de Vries et al. (1997), [3] Dallacasa et al. (2000).

\begin{tabular}{|c|c|c|c|c|c|c|c|c|c|c|c|c|}
\hline Source & id & $m$ & $z$ & $\begin{array}{c}\text { scale } \\
\text { pc/mas }\end{array}$ & $\begin{array}{r}\theta_{\max } \\
\operatorname{mas}\end{array}$ & $\begin{array}{r}l_{\max } \\
\mathrm{pc}\end{array}$ & $\begin{array}{c}S_{\text {merlin }} \\
\mathrm{Jy}\end{array}$ & $\begin{array}{c}S_{1.66} \\
\mathrm{Jy}\end{array}$ & $\alpha_{1}$ & $\alpha_{\mathrm{h}}$ & $\begin{array}{c}\nu_{\mathrm{m}} \\
\mathrm{GHz}\end{array}$ & ref. \\
\hline $1345+125$ & G & $15.5 r$ & 0.122 & 1.5 & 100 & 150 & 4.88 & 4.33 & -0.67 & 0.50 & 0.4 & 1 \\
\hline $1404+286$ & $\mathrm{G}$ & $14.6 r$ & 0.077 & 1.0 & 10 & 10 & 1.14 & 1.00 & -1.5 & 1.6 & 4 & 1 \\
\hline $1518+047$ & $\mathrm{Q}$ & $22.2 R$ & 1.296 & 4.3 & 160 & 688 & 3.55 & 3.00 & -0.20 & 0.93 & 0.9 & 2 \\
\hline $1604+315$ & G & $22.7 r$ & $1.5^{*}$ & 4.3 & $<5$ & $<22$ & 0.83 & 0.75 & -0.59 & 0.28 & 1.5 & 2 \\
\hline \multirow[t]{2}{*}{$1751+278$} & G & $21.7 R$ & $0.86^{*}$ & 4.2 & 50 & 210 & 0.61 & 0.53 & -0.27 & 0.57 & 1.4 & 2 \\
\hline & & & & & & & $S_{2.3}$ & $S_{8.4}$ & & & & \\
\hline $1333+589$ & $\mathrm{EF}$ & & & & 15 & & 0.57 & 0.58 & -0.84 & 0.52 & 4.9 & 3 \\
\hline $1427+109$ & $\mathrm{Q}$ & $18.5 B$ & 1.71 & 4.2 & $<2$ & $<8.4$ & 0.70 & 0.89 & -1.18 & 0.55 & 4.9 & 3 \\
\hline $1509+054$ & G & 16.2 & & & 10 & & 0.20 & 0.71 & -1.72 & 0.46 & 11 & 3 \\
\hline $1526+670$ & $\mathrm{Q}$ & $17.2 E$ & 3.02 & 3.6 & 5 & 18 & 0.25 & 0.28 & -1.26 & 1.21 & 5.8 & 3 \\
\hline $1734+508$ & $\mathrm{G} ?$ & $23.1 R$ & & & 8 & & 0.71 & 0.81 & -0.5 & 0.4 & 5.9 & 3 \\
\hline
\end{tabular}

Table 2. Parameters of the components in the VLBI images at $1.66 \mathrm{GHz}$. The columns give: (1) source name and possible classification (CSO: Compact Symmetric Object; cj: core-jet), (2) component identification, (3) and (4) major and minor axes when a fit has been possible (using JMFIT in AIPS), (5) position angle of the component, (6) and (7) distance and position angle of component with respect to the first component, (8) total flux density of the component, (9) intrinsic brightness temperature neglecting relativistic effects, (10) minimum energy density, (11) equipartition magnetic field.

\begin{tabular}{clccccccccc}
\hline \hline $\begin{array}{c}\text { Source and } \\
\text { class }\end{array}$ & & $\begin{array}{c}\theta_{1} \\
\text { mas }\end{array}$ & $\begin{array}{c}\theta_{2} \\
\text { mas }\end{array}$ & $\begin{array}{c}\text { PA } \\
\circ\end{array}$ & $\begin{array}{c}\mathrm{R} \\
\text { mas }\end{array}$ & $\begin{array}{c}\text { PA } \\
\circ\end{array}$ & $\begin{array}{c}S_{1.66} \\
\mathrm{mJy}\end{array}$ & $\begin{array}{c}T_{\mathrm{b}} \\
10^{9} \mathrm{~K}^{\circ}\end{array}$ & $\begin{array}{c}u_{\text {min }} \\
10^{-6} \mathrm{erg} / \mathrm{cm}^{3}\end{array}$ & $\begin{array}{c}H_{\text {eq }} \\
10^{-3} \mathrm{G}\end{array}$ \\
\hline $1345+125$ & $\mathrm{~A}$ & & & & & & $<40$ & & & \\
CSO & $\mathrm{B}$ & 8 & 1.7 & 136 & 0 & & 302 & 5 & 14 & 12 \\
& $\mathrm{C}$ & 13 & 4.5 & 169 & 8.2 & 138 & 383 & 1.4 & 4 & 7 \\
& $\mathrm{D}$ & & & & & & 3592 & & & \\
$1404+286$ & $\mathrm{NE}$ & 1.9 & 1.2 & 93 & 0 & & 958 & 90 & 100 & 30 \\
$\mathrm{CSO}$ & $\mathrm{SW}$ & 2.7 & 1.3 & 2 & 7.8 & -115 & 14 & 0.8 & 7 & 9 \\
$1518+047$ & $\mathrm{~A}$ & 11 & 3.4 & 33 & 0 & & 1675 & 20 & 35 & 20 \\
$\mathrm{CSO} ?$ & $\mathrm{C}$ & 5.7 & 4.5 & 50 & 136.8 & -153 & 1167 & 20 & 30 & 20 \\
$1604+315$ & & $<5$ & $<5$ & & & & 746 & $>15$ & $>25$ & $>20$ \\
$1751+278$ & $\mathrm{~A}$ & 2.8 & 1 & 92 & 0 & & 479 & 70 & 110 & 35 \\
cj & $\mathrm{B}$ & 3.7 & 3.7 & 1 & 22 & -122 & 39 & 1.0 & 4 & 7 \\
& $\mathrm{C}$ & 17 & 3.2 & 101 & 23 & -120 & 13 & 0.09 & 1.1 & 4 \\
& $\mathrm{D}$ & 17 & 4.6 & 152 & 43 & -94 & 3 & 0.01 & 0.3 & 2 \\
\hline
\end{tabular}

and the lobes $(\mathrm{D}, \mathrm{E})$, while the candidate core $(\mathrm{A})$ is not present, likely self absorbed. For comparison, we convolved the $5 \mathrm{GHz}$ image in Stanghellini et al. (1997a) and the $2.3 \mathrm{GHz}$ image in Fey et al. (1996) with the restoring beam of our $1.66 \mathrm{GHz}$ image, and show the three images in Fig. 1a, superimposed as contours to the same full resolution $5 \mathrm{GHz}$ grey scale image. The image registration has been performed on the basis of the position of the southernmost component, likely free from optical depth effects and with the hypothesis that its proper motion relative to the other components $\mathrm{B}$ and $\mathrm{C}$ is small (like in the hot spots of CSOs).

We find that $\mathrm{A}$ becomes weaker at $2.3 \mathrm{GHz}$ and vanishes at $1.66 \mathrm{GHz}$. We set a very conservative upper limit of $40 \mathrm{mJy}$ for the flux density of this component at $1.66 \mathrm{GHz}$. Component A has therefore an inverted spectrum, is most probably the core and hence $1345+125$ is a CSO. 
Table 3. Parameters of the components in the VLBI images at 2.3 and $8.4 \mathrm{GHz}$. The columns give: (1) source name and possible classification (CSO: Compact Symmetric Object, cj: core-jet; d: double), (2) component identification, (3) total flux density of the component at $2.3 \mathrm{GHz},(4)$ and (5) major and minor axes at $2.3 \mathrm{GHz}$ when fit has been possible (using JMFIT in AIPS), (6) position angle of the component at $2.3 \mathrm{GHz},(7)$ total flux density of the component at $8.4 \mathrm{GHz}$, (8) and (9) major and minor axes at $8.4 \mathrm{GHz},(10)$ position angle of the component at $8.4 \mathrm{GHz},(11)$ and (12) distance and position angle of component with respect to the first component at $8.4 \mathrm{GHz},(13),(14)$ and (15) intrinsic brightness temperature neglecting relativistic effects, minimum energy density, equipartition magnetic field at $8.4 \mathrm{GHz}$.

\begin{tabular}{|c|c|c|c|c|c|c|c|c|c|c|c|c|c|c|}
\hline $\begin{array}{c}\text { Source and } \\
\text { class } \\
\end{array}$ & & $\begin{array}{c}S_{2.3} \\
\mathrm{Jy} \\
\end{array}$ & $\begin{array}{c}\theta_{1} \\
\text { mas }\end{array}$ & $\begin{array}{c}\theta_{2} \\
\text { mas }\end{array}$ & $\begin{array}{c}\text { PA } \\
\circ\end{array}$ & $\begin{array}{c}S_{8.4} \\
\mathrm{Jy}\end{array}$ & $\begin{array}{c}\theta_{1} \\
\text { mas }\end{array}$ & $\begin{array}{c}\theta_{2} \\
\text { mas } \\
\end{array}$ & $\begin{array}{c}\text { PA } \\
\circ\end{array}$ & $\begin{array}{c}\mathrm{R} \\
\text { mas }\end{array}$ & $\begin{array}{c}\mathrm{PA} \\
0\end{array}$ & $\begin{array}{c}T_{\mathrm{b}} \\
10^{9} \mathrm{~K}^{\circ} \\
\end{array}$ & $\begin{array}{c}u_{\min } \\
10^{-6} \frac{\mathrm{erg}}{\mathrm{cm}^{3}}\end{array}$ & $\begin{array}{c}H_{\text {eq }} \\
10^{-3} \mathrm{G}\end{array}$ \\
\hline $1333+589$ & $\mathrm{~N}$ & 0.16 & 4.0 & 1.7 & 153 & 0.42 & 0.5 & $<0.5$ & 177 & 0 & & & & \\
\hline $\mathrm{d} / \mathrm{CSO} ?$ & S & 0.41 & 2.1 & 1.2 & 176 & 0.15 & 1.1 & 0.7 & 39 & 12.8 & -164 & & & \\
\hline $1427+109$ & & 0.70 & $<5$ & $<5$ & & 0.89 & $<2$ & $<2$ & & & & $>5$ & $>330$ & $>60$ \\
\hline $1509+054$ & $\mathrm{E}$ & 0.20 & 4.8 & 1.2 & 109 & 0.3 & 0.8 & $<0.5$ & 98 & 0 & & & & \\
\hline d/CSO? & W & & & & & 0.4 & 0.9 & 0.5 & 94 & 4.9 & -90 & & & \\
\hline $1526+670$ & $\mathrm{E}$ & 0.25 & 1.9 & 1.3 & 82 & 0.1 & 2 & 1 & 45 & 0 & & 1.5 & 530 & 80 \\
\hline cj? & W & & & & & 0.17 & 0.8 & 0.8 & 1 & 0.3 & -126 & 8 & 1600 & 130 \\
\hline $1734+508$ & $\mathrm{~N}$ & 0.70 & 3.1 & 2.4 & 1 & 0.15 & 0.8 & 0.5 & 157 & 0 & & & & \\
\hline $\mathrm{d} / \mathrm{CSO} ?$ & S & & & & & 0.66 & 0.9 & 0.5 & 13 & 2.6 & -144 & & & \\
\hline
\end{tabular}

The source in our MERLIN image seems slightly resolved in the N-S direction (Fig. 1b). A fit with a Gaussian on the image gives a size of about 50 mas. Considering that the angular size measured on an extended region is roughly twice the half maximum width given by the Gaussian fit, it is consistent with the size seen in the EVN image.

\subsection{2. $1404+286(J 1407+2827, O Q 208, M k n 668)$}

The compact radio source at a redshift of 0.077 is one of the closest bright GPS galaxies. With $m_{r}=14.6$, the optical image presented by Stanghellini et al. (1993) shows the presence of companions in the galactic envelope and a tail of low brightness emission in N-S direction, suggesting that the galaxy is dynamically disturbed.

The radio source has been considered in terms of a CSO structure (Stanghellini et al. 2000). It has a weak core at $15 \mathrm{GHz}$, two-sided faint and short jets and double asymmetric mini-lobes located within 10 mas, giving a projected size of less than $10 \mathrm{pc}$. The two micro hot-spots of this radio source seem to increase their distance at a rate of around $0.15 c$, comparable to what already found in other CSOs, yelding a dynamical age of a few centuries (Stanghellini et al. 2000). This radio source has been extensively studied at VLBI resolution, but we included it in our observations at $1.66 \mathrm{GHz}$ because of its remarkable spectral properties at lower frequencies. Actually, Kameno et al. (2000) report a very inverted spectrum below the peak frequency of the weaker south-western region of this source based on VSOP observations. They consider the extremely inverted spectrum inconsistent with synchrotron self-absorption (SSA), and propose a process of free-free absorption (FFA) to explain the radio spectral shape. Our $1.66 \mathrm{GHz}$ image (Fig. 2a) shows two components, a strong NE one (958 mJy) and a weak SW one (14 mJy). Although there is a dirty beam sidelobe close to the position of the southwest component, adding a source of uncertainty to its flux density measure, our estimate of the lobe flux densities from ground VLBI observations is consistent with that of Kameno et al. (2000).

We show the spectra of the two regions resolved by our observations in Fig. 2b where the values at 2.3/8.4 and $5 \mathrm{GHz}$ are from Fey et al. (1996) and Stanghellini et al. (1997b) respectively. We get a slope of the SW component between 1.6 and $2.3 \mathrm{GHz}$ of $\alpha=-3.8 \pm 0.7$, confirming a spectral shape for this component incompatible with simple SSA.

Our MERLIN image gives a pointlike source with no evidence of diffuse emission at the MERLIN scale at a level of $3 \times$ the rms noise of $3 \mathrm{mJy}$.

\subsection{3. $1518+047(J 1521+0430,4 \mathrm{C} 04.51)$}

This object at redshift 1.296 is identified with a quasar of magnitude $m_{R}=22.2$ (Stickel \& Kühr 1996). 1518+047 is a strong radio source with $P_{5 \mathrm{GHz}}=10^{27.9} \mathrm{~W} / \mathrm{Hz}$, and is one of the first examples of a double source at mas resolution. Its morphology is discussed by Mutel et al. (1985), Phillips \& Mutel (1982). They imaged the source with the U.S. VLBI Network at $1.67 \mathrm{GHz}, 5 \mathrm{GHz}$ and $610 \mathrm{MHz}$ (Mutel et al. 1985; Mutel \& Hodges 1986). At $5 \mathrm{GHz}$, the source is resolved into two micro lobes, and each lobe further resolved into two/three components. Dallacasa et al. (1998) also resolve the source in two lobes at $2.3 / 8.4 \mathrm{GHz}$ EVN observations. Our $1.66 \mathrm{GHz}$ EVN image (Fig. 3a) also shows two lobes, with a hint of a jetlike emission (D) associated with the component $\mathrm{C}$, also visible at other frequencies. The resolution of our data did not allow to model components B and D convincingly, but we fitted the main components $\mathrm{A}$ and $\mathrm{C}$ as reported in Table 2. The spectra of the 2 components are pretty similar (Fig. 3b) and this source is very likely a CSO even if a compact core has not been detected yet. The MERLIN image which has a 

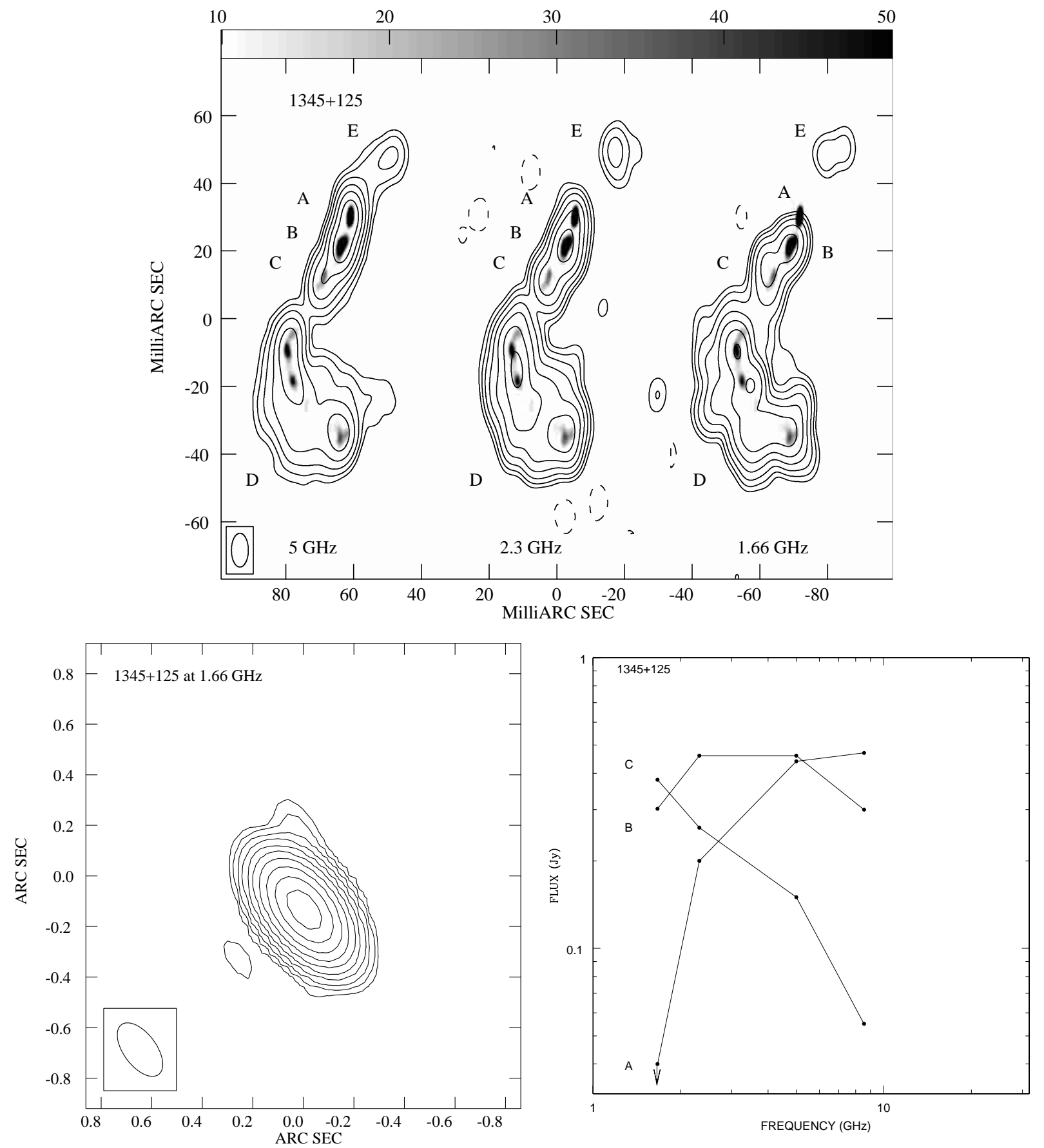

Fig. 1. a) $1345+125$ at $5,2.27,1.66 \mathrm{GHz}$; grey scale image is full resolution $5 \mathrm{GHz}$ image: the restoring beam is $10 \times 5$ mas in $\mathrm{PA} 0^{\circ}$, the rms noise on the image is $2.5 \mathrm{mJy} /$ beam, the contour levels here and in the following figures are $-3,3,6,12,25$, $50,100,200,400,800,1500,3000$ times the rms noise, the peak flux density is $630 \mathrm{mJy} / \mathrm{beam}$. b) $1345+125 \mathrm{MERLIN}$ image at $1.66 \mathrm{GHz}$ : the restoring beam is $256 \times 129$ mas in PA $37^{\circ}$, the rms noise on the image is $2 \mathrm{mJy} /$ beam, the peak flux density is 4236 mJy/beam. c) $1345+125$ : spectra of component A, B and C, data from Stanghellini et al. (1997a), Fey et al. (1996) and this paper.

rms noise of $8 \mathrm{mJy} /$ beam does not reveal any additional emission.

\subsection{4. $1604+315(J 1606+3124)$}

A galaxy with $m_{r}=22.7$ at redshift 1.5 estimated photometrically (as reported by Heckman et al. 1994), is close to the radio position; although very faint, it shows a complex optical morphology elongated in the E-W direction. Other faint objects are present a few arcseconds away, suggesting $1604+315$ is a member of a cluster (Stanghellini et al. 1993). Our VLBI image shows no details (Fig. 4). This source is present in the VLBA calibrator list (Peck et al. 1998) showing a possible core-jet morphology. No extended emission has been detected in our MERLIN image with a rms noise of $1.6 \mathrm{mJy} /$ beam. 

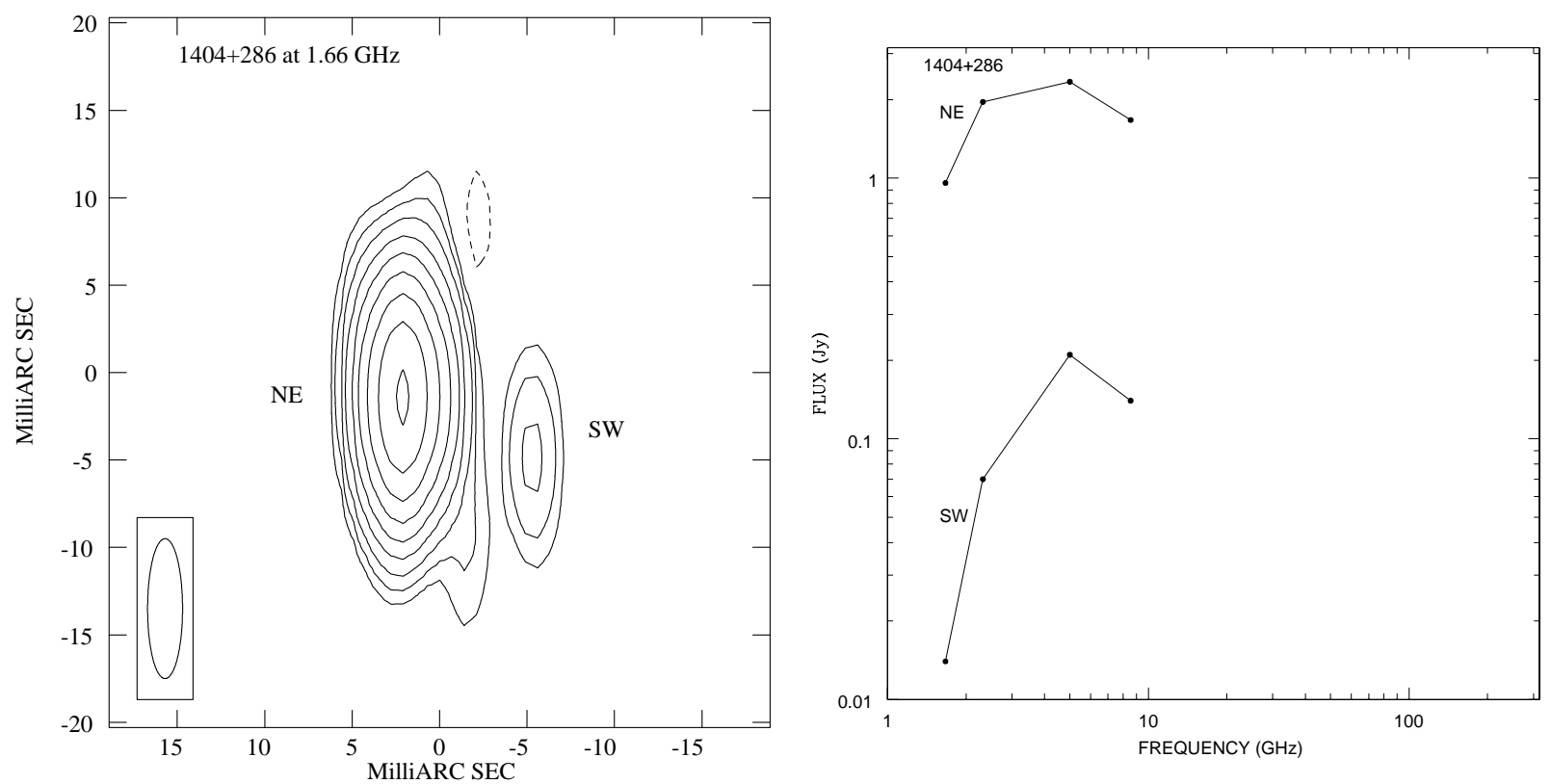

Fig. 2. a) OQ208 EVN image at $1.66 \mathrm{GHz}$ : the restoring beam is $8 \times 2$ mas in PA $0^{\circ}$, the rms noise on the image is $0.5 \mathrm{mJy} / \mathrm{beam}$, the peak flux density is $715 \mathrm{mJy} /$ beam. b) $1404+286$ : spectra of component NE and SW, data from Stanghellini et al. (1997b), Fey et al. (1996) and this paper.
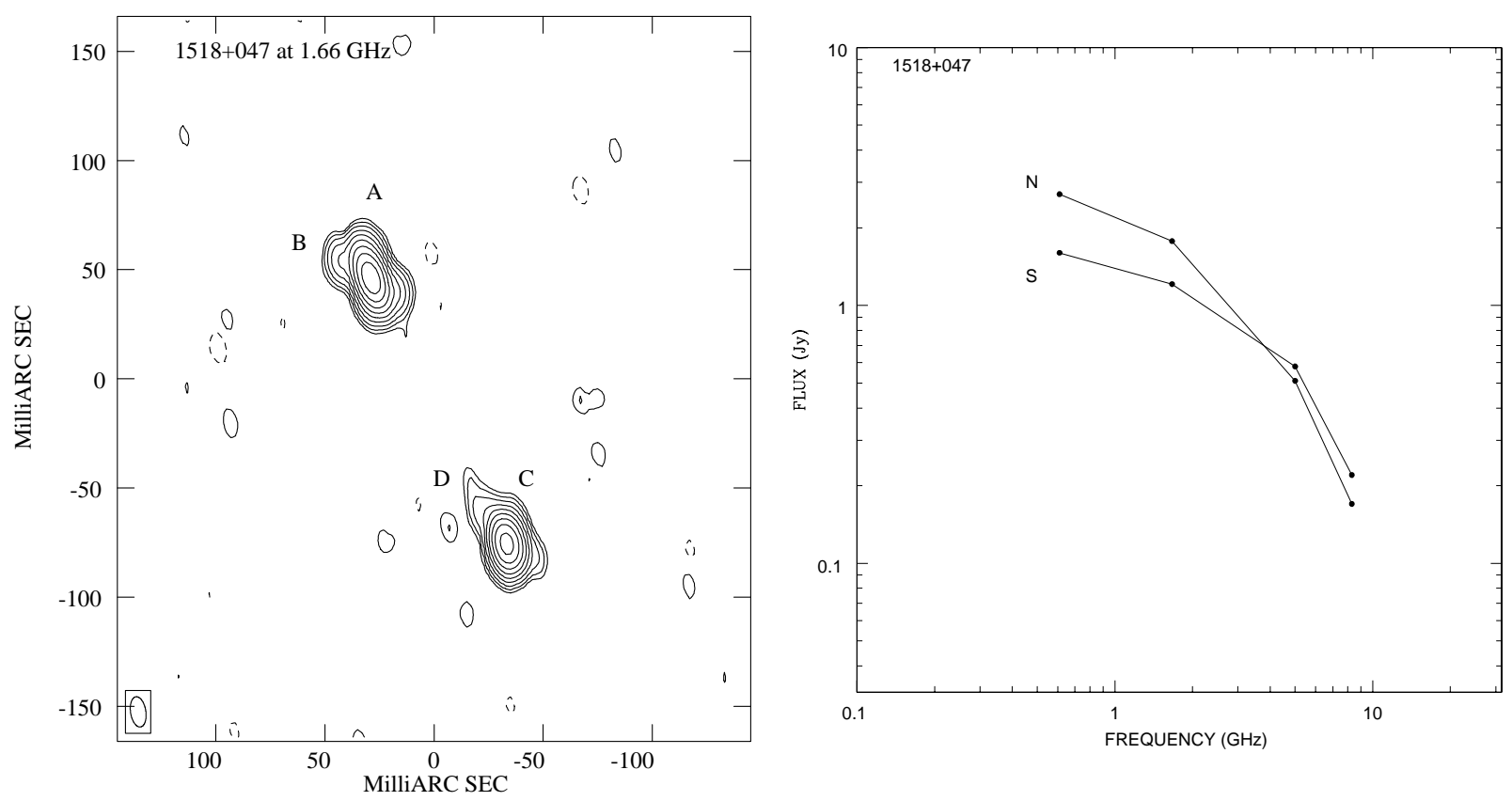

Fig. 3. a) $1518+047 \mathrm{EVN}$ image at $1.66 \mathrm{GHz}$ : the restoring beam is $14 \times 7$ mas in $\mathrm{PA} 8.8^{\circ}$, the rms noise on the image is $1 \mathrm{mJy} /$ beam, the peak flux density is $1144 \mathrm{mJy} /$ beam. b) 1518+047: spectra of component $\mathrm{N}$ and S, data from Mutel et al. (1985), Dallacasa et al. (1998), and this paper. Data at $2.3 \mathrm{GHz}$ from Dallacasa et al. have not been taken in consideration because a significant fraction of the flux density is clearly missing, and were not consistent with the data shown.

\subsection{5. $1751+278(J 1753+2750)$}

This object is identified as a galaxy with $m_{R}=21.7$ and redshift 0.86 estimated photometrically (as reported by Heckman et al. 1994), it is faint and extended (O'Dea et al. 1990), with a possible companion (O'Dea et al. 1996).

No previous VLBI morphology information is available for this source. Our VLBI image at $1.66 \mathrm{GHz}$ (Fig. 5) exhibits a compact dominant component (A), a secondary compact component to south-west (B), from which a trail of radio emission points to west $(\mathrm{C})$, then probably to north-west, where just a hint of emission is visible (D). No extended emission has been detected in our MERLIN image with rms noise of $1.8 \mathrm{mJy} /$ beam. 


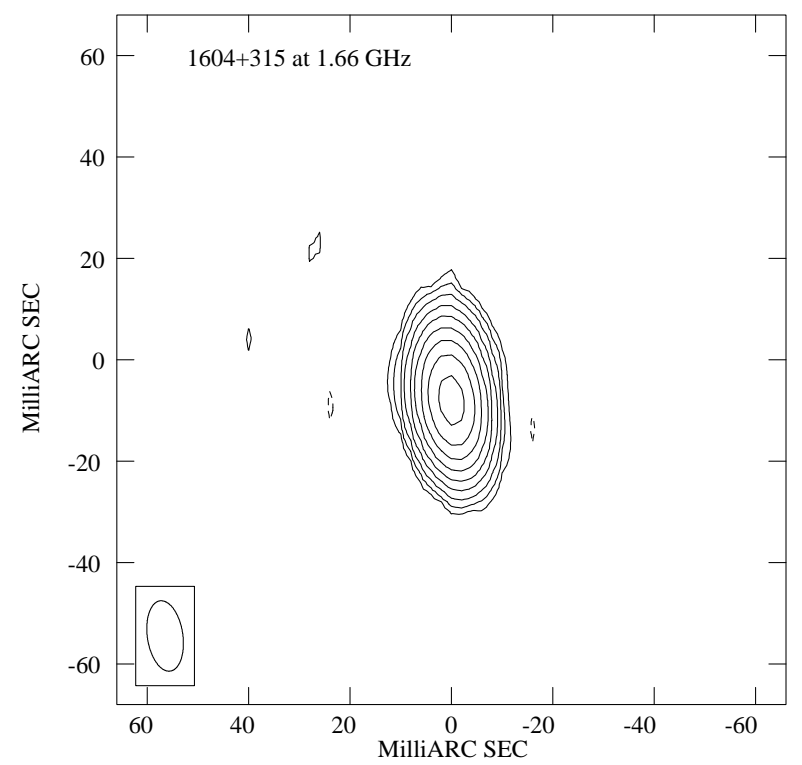

Fig. 4. $1604+315 \mathrm{EVN}$ image at $1.66 \mathrm{GHz}$ : the restoring beam is $14 \times 7$ mas in PA $8.4^{\circ}$, the rms noise on the image is $0.6 \mathrm{mJy} /$ beam, the peak flux density is $662 \mathrm{mJy} /$ beam.

This source is classified as a GPS source on the basis of a flux density limit of $0.25 \mathrm{Jy}$ at $408 \mathrm{MHz}$ (Spoelstra et al. 1985). In the literature three values of the flux density of this source at $1.4 \mathrm{GHz}$ are found, which are $0.52 \pm 0.06 \mathrm{Jy}$ (Condon et al. 1983, and references therein), $0.647 \pm 0.030 \mathrm{Jy}$ (White et al. 1992), and $0.6253 \pm 0.0188 \mathrm{Jy}$ (NVSS value). The flux density at $365 \mathrm{MHz}$ is $0.685 \pm$ 0.069 Jy (Douglas et al. 1996), so the flux density limit at $408 \mathrm{MHz}$ seems wrong, (or the source is highly variable at low frequency). Given the spectral flattening of this source above $1.6 \mathrm{GHz}$, the lack of a peak in the spectrum, and its possible variability, the source could be a flat spectrum or a compact steep spectrum (CSS), rather than a GPS radio source and we will not consider it in the discussion of the results.

\subsection{Images at 2.3 and $8.4 \mathrm{GHz}$}

\subsection{1. $1333+589(J 1335+5844,4$ C58.26)}

This radio source has a peak flux density of $0.75 \mathrm{Jy}$ at $4.9 \mathrm{GHz}$; in the optical it is an empty field (Stickel \& Kühr 1994). This object belongs to the CJ1 sample (the first Caltech-Jodrell Bank survey), and has been already imaged with VLBI at $1.67 \mathrm{GHz}$ (Thakkar et al. 1995) and $5 \mathrm{GHz}$ (Xu et al. 1995). The source is clearly resolved into two compact components at both forementioned frequencies, and this morphology is confirmed in our $2.3 / 8.4 \mathrm{GHz}$ images. As shown in Figs. 6a and 6b, the two components are separated by $12.8 \pm 0.1$ mas, and no jetlike feature has been detected between them. We show in Fig. $6 c$ the spectra of the components using our images and the data at 1.67 and $5 \mathrm{GHz}$ mentioned above.

The northern component show a rising spectrum at all frequencies, indicating it is more compact. It is possible

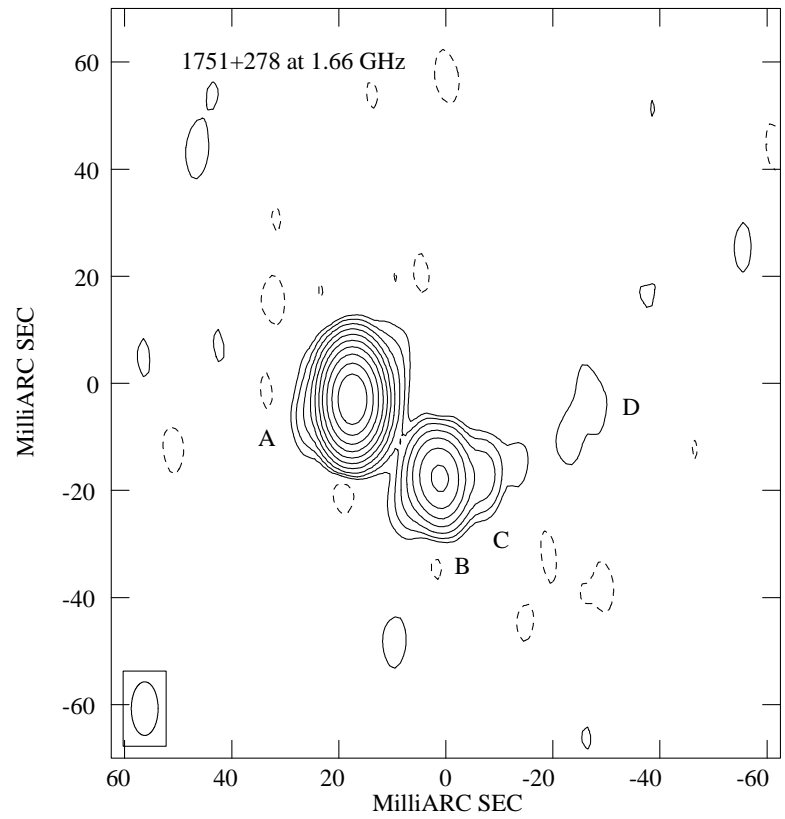

Fig. 5. $1751+278 \mathrm{EVN}$ image at $1.66 \mathrm{GHz}$ : the restoring beam is $10 \times 5$ mas in PA $0^{\circ}$, the rms noise on the image is $0.2 \mathrm{mJy} /$ beam, the peak flux density is $417 \mathrm{mJy} /$ beam.

that it is the core of the radio emission and this object being a core-jet radio source. Indeed this source is not considered a CSO in Taylor (1996), Polatidis et al. (1999), Peck \& Taylor (2000), who have examined all candidates in the PR, CJ1 and CJ2 surveys with multi-frequency VLBA observations. We think it is also possible that the rising spectrum reflects a very compact micro-hotspot, smaller than in the southern lobe, and the radio source still would be a CSO.

More sensitive high resolution observations to detect any further emission should provide a better constraint for the correct morphological classification.

\subsection{2. $1427+109(\mathrm{~J} 1430+1043)$}

The optical host is a quasar at $z=1.710$ and magnitude $m_{B}=18.5$ (NASA/IPAC Extragalactic Database). In the radio band this source peaks at $4.9 \mathrm{GHz}$ with a flux density of $0.91 \mathrm{Jy}$. The source is not resolved in our $2.3 / 8.4 \mathrm{GHz}$ images (not shown), and no previous VLBI morphology information is available in the literature.

\subsection{3. $1509+054(\mathrm{~J} 1511+0518)$}

The optical identification for this radio source is a galaxy with visual magnitude 16.2 (Dallacasa et al. 2000). The source shows a very steep spectrum with a peak flux density of $0.77 \mathrm{Jy}$ at $11 \mathrm{GHz}$. Our $8.4 \mathrm{GHz}$ image (Fig. 7) shows two components separated by 4.9 mas; the resolution at $2.3 \mathrm{GHz}$ does not allow to separate these two components. No VLBI image is available in the literature for this source. The double morphology at $8.4 \mathrm{GHz}$ makes this source a CSO candidate. 

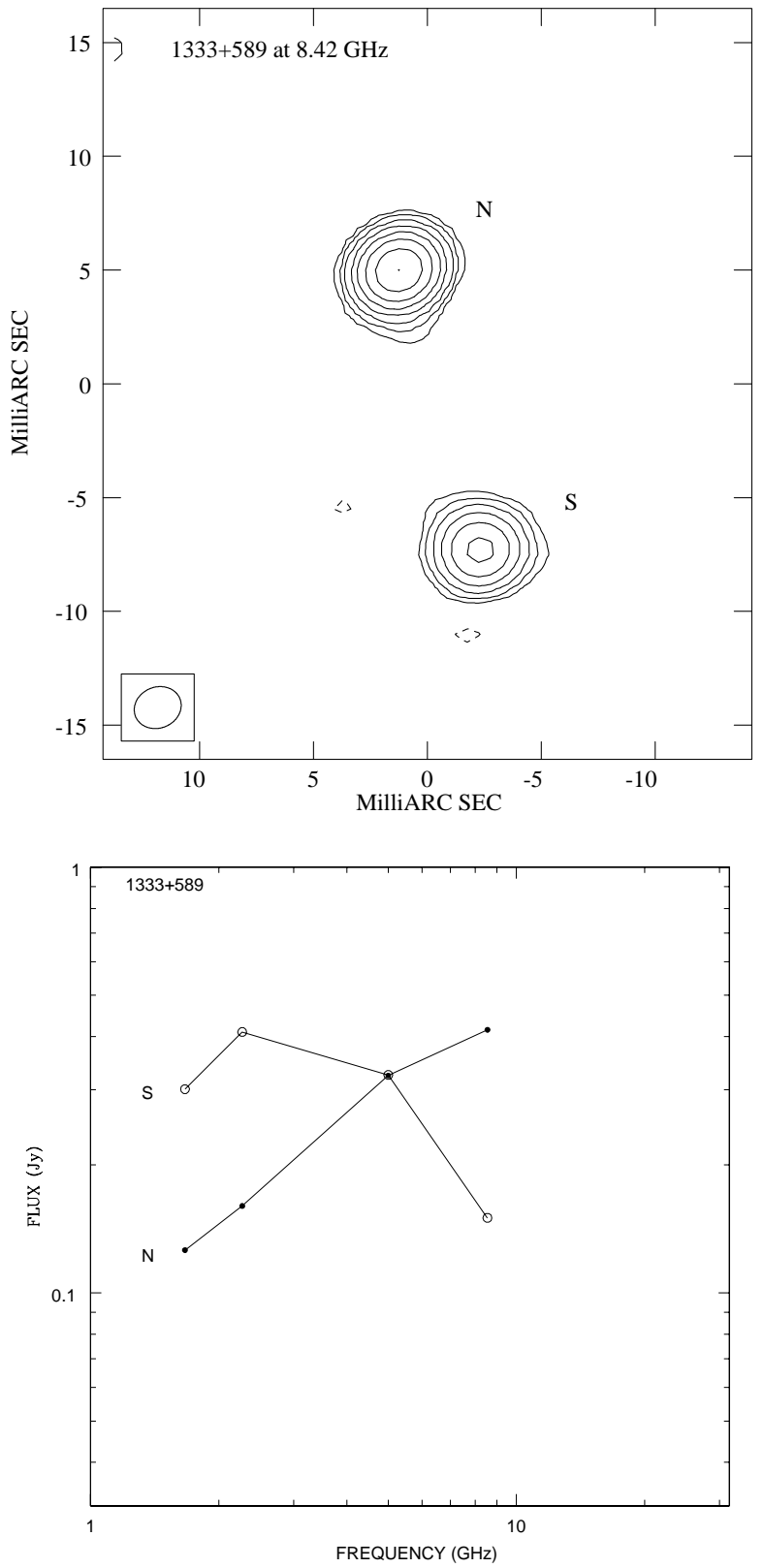

3.2.4. $1526+670(\mathrm{~J} 1526+6650)$

The host of the source is a quasar at high redshift $(z=$ 3.02) and magnitude $m_{r}=17.2$ (Hook et al. 1996). This is a CJ2 source and has been imaged at $5 \mathrm{GHz}$ (Taylor et al. 1994). It is slightly elongated in our $8.4 \mathrm{GHz}$ image (Fig. 8) in agreement with the $5 \mathrm{GHz}$ image, and the radio emission can be modelled with two components as listed in Table 3. This object is unresolved in our $2.3 \mathrm{GHz}$ image.

The spectrum of the components between $8.4 \mathrm{GHz}$ and $5 \mathrm{GHz}$ (Taylor et al. 1994), shows that the western, brighter component has a flatter spectral index than the eastern component, hence it is possible that this source is a core-jet object, but new observations at higher frequency and resolution are necessary to give a proper classification.

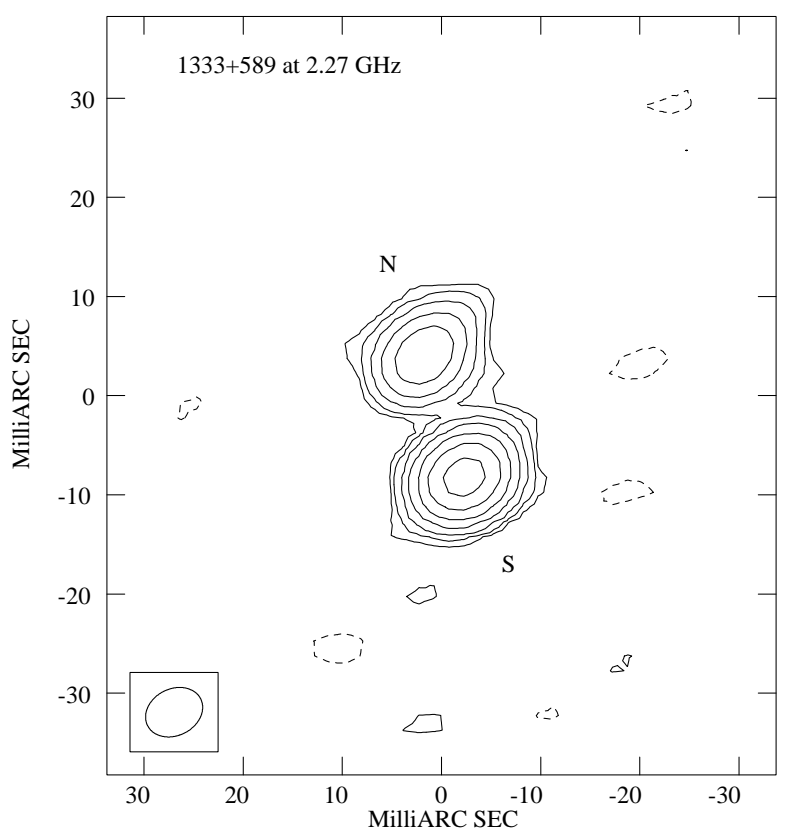

Fig. 6. a) $1333+589 \mathrm{EVN}$ image at $8.4 \mathrm{GHz}$ : the restoring beam is $2.1 \times 1.8$ mas in $\mathrm{PA} 112^{\circ}$, the rms noise on the image is $1 \mathrm{mJy} /$ beam, the peak flux density is $401 \mathrm{mJy} / \mathrm{beam}$. b) $1333+589 \mathrm{EVN}$ image at $2.3 \mathrm{GHz}$ : the restoring beam is $6 \times$ 4.7 mas in PA $117^{\circ}$, the rms noise on the image is $1.3 \mathrm{mJy} /$ beam, the peak flux density is $480 \mathrm{mJy} /$ beam. c) 1333+589: spectra of component N, S and N+S, data from Xu et al. (1995), Thakkar et al. (1995) and this paper.

\subsection{5. $1734+508(J 1735+5049)$}

A very faint $\left(m_{R}=23.1\right)$ optical object has been detected at the radio position, and tentatively classified as a galaxy (Stickel \& Kühr 1996). The radio source peaks at 5.9 GHz with a peak flux density of $0.99 \mathrm{Jy}$. This is a CJ1 source, resolved in two components at $5 \mathrm{GHz}$, and modelled with three Gaussian components (Xu et al. 1995), Our 8.4 GHz image (Fig. 9) shows a double morphology, while it is unresolved in our image at 2.3 , as at $1.6 \mathrm{GHz}$ (Thakkar et al. 1995). Considering the model given by $\mathrm{Xu}$ et al. at $5 \mathrm{GHz}$, the northern component has a steep spectrum $(\alpha \sim 0.8)$, while the southern one is slightly inverted $(\alpha \sim-0.2)$. As for $1333+589$ this source has not been considered a CSO 


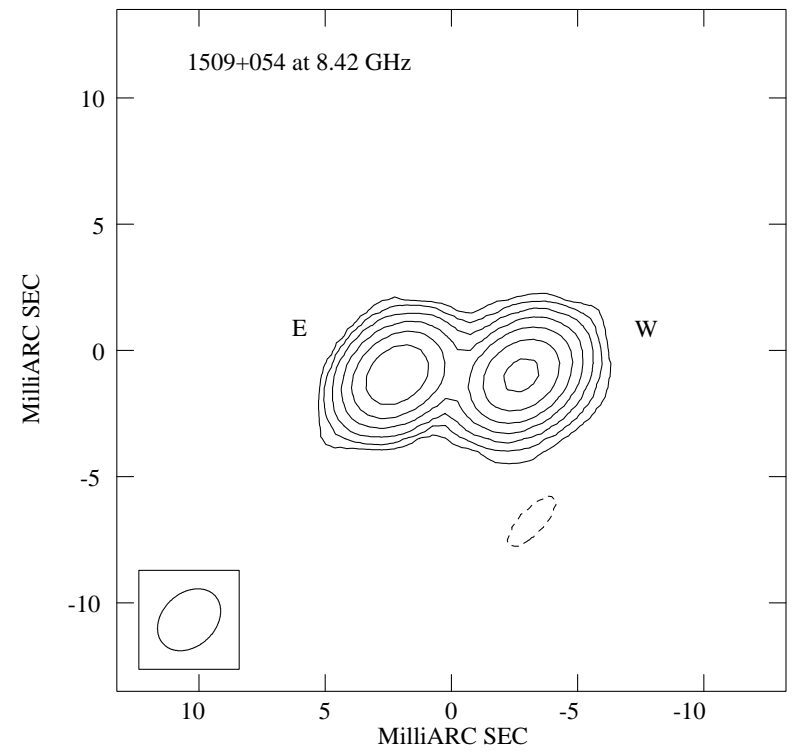

Fig. 7. $1509+054 \mathrm{EVN}$ image at $8.4 \mathrm{GHz}$ : the restoring beam is $2.8 \times 2.1$ mas in $\mathrm{PA} 133^{\circ}$, the rms noise on the image is $1.5 \mathrm{mJy} /$ beam, the peak flux density is $374 \mathrm{mJy} /$ beam.

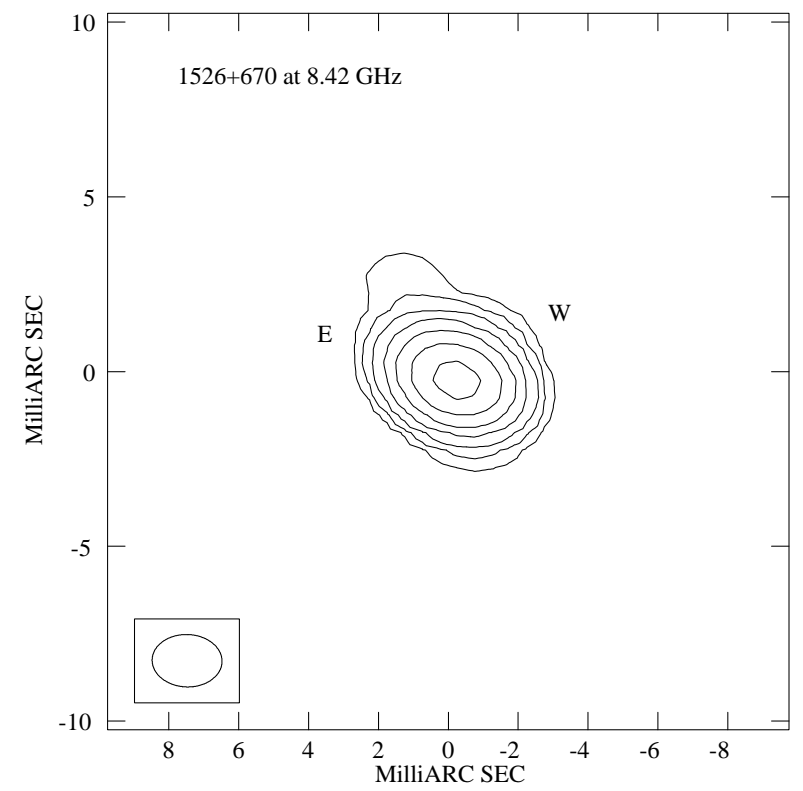

Fig. 8. $1526+670 \mathrm{EVN}$ image at $8.4 \mathrm{GHz}$ : the restoring beam is $2 \times 1.5$ mas in PA $88^{\circ}$, the rms noise on the image is $0.8 \mathrm{mJy} /$ beam, the peak flux density is $216 \mathrm{mJy} /$ beam.

by Taylor (1996), Polatidis et al. (1999), or Peck \& Taylor (2000). Also in this case we think it is possible that an inverted spectrum is consistent either with a core or a very compact hot-spot. Until additional high resolution morphological information will be available we consider this source a CSO candidate, given its double morphology.

\section{Discussion and conclusions}

We can summarize the results from our EVN observations at $1.66,2.3$ and $8.4 \mathrm{GHz}$ for $10 \mathrm{GPS}$ radio sources as follows:

i) We confirm the CSO nature of $1345+125$ revealing a component which is very likely to be the core.

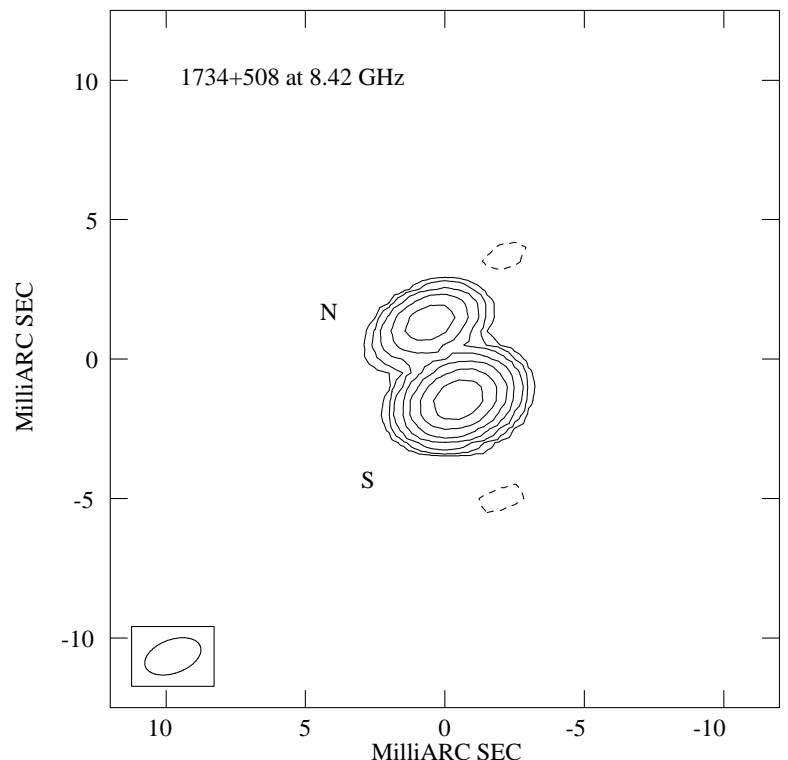

Fig. 9. $1734+508 \mathrm{EVN}$ image at $8.4 \mathrm{GHz}$ : the restoring beam is $2.1 \times 1.2$ mas in $\mathrm{PA} 110^{\circ}$, the rms noise on the image is $1.5 \mathrm{mJy} /$ beam, the peak flux density is $528 \mathrm{mJy} /$ beam.

ii) For the radio source $1404+286$ we confirm from ground based VLBI observations the findings by Kameno et al. (2000) i.e. that the radio spectrum is affected by FFA rather than SSA, or maybe both. This is an important observational clue because it implies the presence of a dense ionized ambient medium somewhere between us and the strongly absorbed radio emitting region.

iii) We also found more CSO candidates which need need further observations to be confirmed.

The relationship between radio mas morphology and optical host is rather respected in the small sample considered here, although also quasars may appear in the CSO candidate list (e.g. 1518+047). Excluding 1751+278 which does not show a convex radio spectrum and it has been erroneously classified as a GPS radio source, we find that 5 galaxies (we include $J 1335+5844$ in the galaxy group as empty fields are mostly identified with weak galaxies once sensitive optical observations become available) are confirmed or candidate CSO, one quasar is also a CSO candidate and 2 quasars are unresolved or slightly resolved objects in our images. Therefore the observations of GPS radio galaxies seem to be an efficient way to find CSOs.

The MERLIN data at $1.66 \mathrm{GHz}$ do not reveal any extended emission in four of the five sources observed. Only $1345+125$ is very sligthly resolved, in agreement with the size seen in the EVN image.

Since the cores are known to have highly inverted spectra, multi-frequency and especially high frequency sensitive observations are needed to confirm the CSO nature for the new CSO candidates discovered here. If they are confirmed they will be very suitable objects to monitor for separation speed, due to their small size (thus possibly faster separation velocity) and the presence of strong and compact components (hot-spots?) at their edges. 
Acknowledgements. Part of this work was supported by the National Natural Science Foundation of China. L.X. wish to thank finacial supports from the CNR Istituto di Radioastronomia (Italy) and the Joint Institute of VLBI in Europe (in The Netherlands) for the work.

\section{References}

Condon, J. J., Condon, M. A., Broderick, J. J., \& Davis, M. M. 1983, AJ, 88, 20

Dallacasa, D., Stanghellini, C., Centonza, M., \& Fanti, R. 2000, A\&A, 363, 887

Dallacasa, D., Bondi, M., Alef, W., \& Mantowani, F. 1998, A\&AS, 129, 219

de Vries, W. H., Barthel, P. D., \& O'Dea, C. P. 1997, A\&AS, 321,105

Douglas, J. N., Bash, F. N., Bozyan, F. A., Torrence, G. W., \& Wolfe, C. 1996, AJ, 111, 1945

Evans, A. S., Kim, D. C., Mazzarella, J. M., et al. 1999, ApJ, 521, L107

Fanti, C., Fanti, R., Dallacasa, D., et al. 1995, A\&A, 302, 317

Fanti, C. 2000, Proceedings of the 5th European VLBI Network Symposium (2000), held at Chalmers Technical University, June 29th-July 1st, 2000. ed. J. E. Conway, A. G. Polatidis, R. S. Booth, \& Y. Pihlström (Published Onsala Space Observatory), ISBN 91-631-0548-9

Fey, A. L., Clegg, A. W., \& Fomalont, E. B. 1996, ApJS, 105, 299

Heckman, T. M., Smith, E. P., Baum, S. A., et al. 1986, ApJ, 311,526

Heckman, T. M., O'Dea, C. P., Baum, S. A., \& Laurikainen, E. 1994, ApJ, 428, 65

Hook, I. M., McMahan, R. G., Irwin, M. J., \& Hazard, C. 1996, MNRAS, 282, 1274

Kameno, S., Horiuchi, S., Shen, Z.-Q., Inoue, M., \& Kobayashi, H. 2000, PASJ, 52, 209

Mutel, R. L., Hodges, M. W., \& Phillips, R. B. 1985, ApJ, 290, 86

Mutel, R. L., \& Hodges, M. W. 1986, ApJ, 307, 472

O'Dea, C. P., Baum, S. A., \& Morris, G. B. 1990, A\&AS, 82, 261

O'Dea, C. P., Baum, S. A., \& Stanghellini, C. 1991, ApJ, 380, 66

O’Dea, C. P., Stanghellini, C., Baum, S. A., \& Charlot, S. 1996, ApJ, 470, 806

Owsianik, I., Conway, J. E., \& Polatidis, A. G. 1998, A\&A, 336, L37

Owsianik, I., \& Conway, J. E. 1998, A\&A, 337, 69

Peck, A. B., \& Beasley, A. J. 1998, in Radio Emission from Galactic and Extragalactic Compact Sources, 5ASP Conf. Ser., 144, IAU Colloq. 164, ed. J. A. Zensus, G. B. Taylor, \& J. M. Wrobel, 155
Peck, A. B., \& Taylor, G. B. 2000, ApJ, 534, 90

Phillips, R. B., \& Mutel, R. L. 1982, A\&A, 106, 21

Polatidis, A., Wilkinson, P. N., Xu, W., et al. 1999, NewAR, 43,657

Readhead, A. C. S., Taylor, G. B., Xu, W., Pearson, T. J., \& Wilkinson, P. N. 1996a, ApJ, 460, 612

Readhead, A. C. S., Taylor, G. B., Pearson, T. J., \& Wilkinson, P. N. 1996b, ApJ, 460, 634

Shepherd, M. C. 1997, in Astronomical Data Analysis Software and Systems VI, ed. G. Hunt, \& H. E. Payne, ASP, Conf. Ser., 125

Snellen, I. A. G., Schilizzi, R. T., Bremer, M. N., et al. 1999, MNRAS, 307, 149

Snellen, I. A. G., Schilizzi, R. T., Miley, G. K., et al. 2000, MNRAS, 319, 445

Spoelstra, T. A. T., Patnaik, A. R., \& Gopal-Krishna 1985, A\&A, 152, 38

Stanghellini, C., O'Dea, C. P., Baum, S. A., \& Laurikainen, E. 1993, ApJS, 88, 1

Stanghellini, C., O'Dea, C. P., Baum, S. A., et al. 1997a, A\&A, 325,943

Stanghellini, C., Bondi, M., Dallacasa, D., et al. 1997b, A\&A, 318,376

Stanghellini, C., O’Dea, C. P., Dallacasa, D., et al. 1998, A\&AS, 131, 303

Stanghellini, C., Dallacasa, D., Bondi, M., \& Liu Xiang 2000, Proceedings of the 5th European VLBI Network Symposium (2000), held at Chalmers Technical University, June 29th-July 1st, 2000, ed. A. G. Polatidis, R. S. Booth, \& Y. Pihlström (Published Onsala Space Observatory), ISBN 91-631-0548-9

Stickel, M., \& Kühr, H. 1994, A\&AS, 103, 349

Stickel, M., \& Kühr, H. 1996, A\&AS, 115, 11

Taylor, G. B., Vermeulen, R. C., Pearson, T. J., et al. 1994, ApJS, 95, 345

Taylor, G. B. 1996, in Proceedings of the Second Workshop on Gigahertz Peaked Spectrum and Compact Steep Spectrum Radio Sources, ed. I. A. G. Snellen, R. T. Schilizzi, H. J. A. Röttgering, \& M. N. Bremer (Leiden, Leiden Observatory), 263

Thakkar, D. D., Xu, W., Readhead, A. C. S., et al. 1995, ApJS, 98, 33

White, R. L., \& Becker, R. H. 1992, ApJS, 79, 331

Wilkinson, P. N., Polatidis, A. G., Readhead, A. C. S., Xu, W., \& Pearson, T. J. 1994, ApJ, 432, L87

Xu, W., Readhead, A. C. S., Pearson, T. J., Polatidis, A. G., \& Wilkinson, P. N. 1995, ApJS, 99, 297

Zhang, H. Y., Liu, X., Jin, C. J., \& Nan, R. D. 2001, ChJAA, 1,129 